OPEN ACCESS

Check for updates

\section{Multifactorial falls prevention programme compared with usual care in UK care homes for older people: multicentre cluster randomised controlled trial with economic evaluation}

\author{
Pip A Logan, 1,2,3,4 Jane C Horne, ${ }^{1}$ John R F Gladman, 1,3,4,5 Adam L Gordon, 1,3,4,6 Tracey Sach, \\ Allan Clark, ${ }^{7}$ Katie Robinson, ${ }^{1,5}$ Sarah Armstrong, ${ }^{1}$ Sue Stirling, ${ }^{7}$ Paul Leighton, ${ }^{1}$ Janet Darby, ${ }^{1}$ \\ Fran Allen, ${ }^{1}$ Lisa Irvine, ${ }^{8}$ Ed C F Wilson, ${ }^{7}$ Chris Fox, ${ }^{9}$ Simon Conroy, ${ }^{10}$ Gail Mountain, ${ }^{11}$ \\ Karen McCartney, ${ }^{1}$ Maureen Godfrey, ${ }^{12}$ Erika Sims $^{7}$
}

For numbered affiliations see end of the article

Correspondence to: P A Logan pip.logan@nottingham.ac.uk (or @loganpip on Twitter: ORCID 0000-0002-6657-2381) Additional material is published online only. To view please visit the journal online.

Cite this as: BMJ 2021;375:e066991 http://dx.doi.org/10.1136/ bmj-2021-066991

Accepted: 12 November 2021

\section{ABSTRACT}

\section{OBJECTIVES}

To determine the clinical and cost effectiveness of a multifactorial fall prevention programme compared with usual care in long term care homes.

DESIGN

Multicentre, parallel, cluster randomised controlled trial.

\section{SETTING}

Long term care homes in the UK, registered to care for older people or those with dementia.

\section{PARTICIPANTS}

1657 consenting residents and 84 care homes. 39 were randomised to the intervention group and 45 were randomised to usual care.

INTERVENTIONS

Guide to Action for Care Homes (GtACH): a multifactorial fall prevention programme or usual care.

\section{MAIN OUTCOME MEASURES}

Primary outcome measure was fall rate at 91-180 days after randomisation. The economic evaluation measured health related quality of life using quality adjusted life years (QALYS) derived from the five domain five level version of the EuroQoL index (EQ-5D$5 \mathrm{~L}$ ) or proxy version (EQ-5D-5L-P) and the Dementia Quality of Life utility measure (DEMQOL-U), which were self-completed by competent residents and by a care home staff member proxy (DEMQOL-P-U) for

\section{WHAT IS ALREADY KNOWN ON THIS TOPIC}

Falls are common in older residents of care homes and are associated with high risk of injury, admissions to hospital, and important cost to healthcare systems Although interventions for falls prevention have been shown to be effective in other settings, previous systematic reviews suggested that the benefits were uncertain in care home residents

Interventions in care homes tend to be more effective if they are co-designed with residents and staff and take account of the care home context

\section{WHAT THIS STUDY ADDS}

A multifactorial falls prevention programme in care homes for older people, codesigned with care home staff and residents, that involved awareness raising, education, screening, decision support, and implementation support was associated with a reduction in falls rate

The intervention was found to be cost effective

No adverse effects of the intervention were found on residents' activity levels or physical dependency all residents (in case the ability to complete changed during the study) until 12 months after randomisation. Secondary outcome measures were falls at 1-90, 181-270, and 271-360 days after randomisation, Barthel index score, and the Physical Activity MeasureResidential Care Homes (PAM-RC) score at 91, 180, 270 , and 360 days after randomisation.

\section{RESULTS}

Mean age of residents was 85 years. $32 \%$ were men. $\mathrm{GtACH}$ training was delivered to $1051 / 1480$ staff (71\%). Primary outcome data were available for 630 participants in the GtACH group and 712 in the usual care group. The unadjusted incidence rate ratio for falls between 91 and 180 days was 0.57 (95\% confidence interval 0.45 to $0.71, \mathrm{P}<0.001$ ) in favour of the GtACH programme (GtACH: six falls $/ 1000$ residents $v$ usual care: 10 falls/1000). Barthel activities of daily living indices and PAM-RC scores were similar between groups at all time points. The incremental cost was $f 108$ (95\% confidence interval $-£ 271.06$ to 487.58$)$, incremental QALYs gained for EQ-5D-5L-P was 0.024 (95\% confidence interval 0.004 to 0.044$)$ and for DEMQOL-P-U was $0.005(-0.019$ to 0.03$)$. The incremental costs per EQ-5D-5L-P and DEMQOL-P-U based QALY were $\mathrm{f} 4544$ and $£ 20889$, respectively.

\section{CONCLUSIONS}

The GtACH programme was associated with a reduction in fall rate and cost effectiveness, without a decrease in activity or increase in dependency.

\section{TRIAL REGISTRATION}

ISRCTN34353836.

\section{Introduction}

Falls are three times more common in care home residents than people of similar age living in the community. ${ }^{1}$ In the United Kingdom, around $4 \%$ of those older than 65 years and $15 \%$ of those older than 85 years live in care homes that provide 24 hour care with or without nursing input. ${ }^{2}$ Falls are a problem in the current population of 400000 care home residents living in the UK, most of whom are at high risk of falling. This poses a high cost to society and adds burden to health and social care systems, such as ambulance services and emergency departments. Falls are associated with personal cost to residents and their families, with some residents never returning to their previous level of function. Most residents have multiple medical conditions and limitations in activities of daily 
living. ${ }^{3}$ Serious injuries account for around $25 \%$ of falls in care home residents, and $40 \%$ of admissions from care home to hospital are related to falls. ${ }^{45}$ A systematic review of the randomised controlled trial evidence in 2018 concluded that effective interventions to prevent falls in community dwelling people (exercise, drug review, and multifactorial interventions) were of uncertain benefit in care home residents. ${ }^{6}$ Given the multiple intrinsic and extrinsic risk factors for falls in care homes, the review advised further evaluation of multifactorial interventions.

Using a co-design approach, our research group developed and tested in a feasibility randomised controlled trial, an intervention programme to prevent falls in care home residents, the Guide to Action Care Home (GtACH) programme. ${ }^{78}$ These reported results provided the evidence and justification to conduct a full definitive trial. The multi-domain GtACH programme includes one hour of training for all care home staff (including gardeners, caretakers, cooks, cleaners, managers) in small groups, delivered by a falls specialist. After training, a manual summarising the GtACH programme and including resources such as a falls incident chart (to detect patterns) and a drug falls risk chart is left in the care home. Once trained, staff are expected to use the GtACH risk assessment and checklist for all residents. For example, the assessment might highlight that a resident is dehydrated, and the recommended action is to increase fluid intake. The manual and training prompts care home staff to take action for the dehydration, such as introducing smoothies, offering fruit juice more often, adapting crockery to take account of disabilities, producing soups, and making an event of coffee time. Overall, the training and resources increase both awareness and knowledge about the management of falls. In our multicentre randomised controlled trial (the Falls in Care Homes (FinCH) study), we determined the clinical and cost effectiveness of the GtACH programme compared with usual care in UK care homes.

\section{Methods}

We performed a multicentre, parallel, 1:1 cluster randomised controlled trial to evaluate the GtACH programme compared with usual care for the prevention of falls in older residents of long term care homes in the UK; the full protocol has been published. ${ }^{9}$ The primary health outcome was fall rate 91-180 days after randomisation of the care home. This timeframe was chosen to allow time to train and embed the use of the programme, as determined by the feasibility study. ${ }^{8}$ A within trial economic evaluation estimated the cost effectiveness of the GtACH programme from a health and personal social services perspective, as recommended. ${ }^{10}$ This trial was designed as near to real life as possible, such that the percentage of care home staff trained to use the GtACH programme in the intervention homes was collected but the number of actions to prevent falls, such as new spectacles, was not collected. Our process evaluation completed in six homes will be presented in a separate paper.

\section{Care home and participant recruitment}

Sites comprised National Health Service organisations with established community based falls teams that were willing to participate in the trial, and the care homes in geographical areas covered by the corresponding NHS Clinical Commissioning Group or Integrated Care System. Sites were identified through the National Institute of Health Research Clinical Research Network, which aims to connect research teams with relevant clinical services. Ten sites across England recruited eligible care homes. To be eligible the homes had to be long stay and registered to care for older people or those with dementia, have 10 or more residents, routinely record falls, and have the agreement of the care home owner. Care homes were not eligible if they had participated in previous GtACH studies, ${ }^{8}$ provided care for those with learning difficulties, did not agree to the intervention being used, or were under special measures from the UK national regulator of care homes (the Care Quality Commission). Most care homes in the UK are privately owned and none are provided by the NHS. They are paid for by residents, or by the state if residents are unable to pay. The NHS provide healthcare to residents as if they were living in their own homes-for example, each resident is registered with a local general practitioner. Owners of care homes employ a range of professionally registered healthcare workers, such as nurses and unregistered care staff, depending on their licence.

Care homes were identified from the register of care homes held by the Care Quality Commission; contacted by email, telephone, and letter; and recruited by research staff in person. At this meeting the researcher explained the study, ensured that falls were being recorded in the personal care records and on the falls incident sheets according to the recommendations of the Care Quality Commission, and used the same definition of a fall-an unintentional or unexpected loss of balance resulting in coming to rest on the floor, the ground, or an object below knee level. ${ }^{10}$

All residents in the recruited homes were eligible to participate, including those who lacked mental capacity to provide consent, except for residents who the care home staff determined to be in the last few days of life or who were receiving short term care or rehabilitation. A member of the research team approached and recruited residents with mental capacity. Residents lacking mental capacity were recruited through personal (family or friends) or professional (care home manager) consultees. The time between the first participant recruited and randomisation was four weeks.

\section{Randomisation and blinding to allocation}

Randomisation took place four weeks after recruitment of the first resident in a participating home, as our previous feasibility showed that little additional recruitment was possible after this time. Site trial coordinators randomised care homes on a 1:1 basis to one of two parallel arms (the GtACH programme or usual care) using a bespoke computer generated pseudo- 
random code of variable block randomisation within strata (site, care home type (nursing, residential, dual registration)) provided by the Norwich Clinical Trials Unit through a secure web based randomisation service. Control homes were offered the intervention at the end of the trial. The researchers, resident participants, and staff informants were blind to allocation at consent and to baseline data collection. Researchers collecting data remained blind to allocation but documented if they became unblinded. By the nature of the intervention, care home staff and resident participants could not be blind to allocation group. All Hospital Episode Statistics data were extracted and analysed blind to allocation. The data monitoring committee were not blinded to the allocation for safety events. Treatment allocations were concealed from the study statistician until the main analyses were complete.

\section{Intervention}

The published GtACH programme ${ }^{7}{ }^{8}$ is described in full detail using the headings from the Template for Intervention Description and Replication (TIDiER) checklist and a full TIDiER checklist in a supplementary file (supplementary materials S1). ${ }^{11}$

\section{Rationale}

Owing to care home staff being relatively untrained, the complex nature of risk factors for falls in care residents, and the need for several interventions to deal with multiple risk factors, a systematic care home wide programme including staff education and support in the use of risk assessment and decision support tools was required. The GtACH programme is a systematic approach developed using literature, clinical expertise, and the views of care home residents and families, care home staff, and researchers. The theory was that staff are key to reducing fall rate in care facilities and that by numerous incremental actions, such as improved lighting, greater access to appropriate drinks, timely drug reviews, and monitoring the pattern of falls then the effect on an individual will be seen.

\section{GtACH programme}

A power point presentation was used in the training. Care home staff received a manual, a paper falls screening and assessment tool, a paper Falls Incident Analysis template, a drug and falls chart, training attendance certificate, and poster to remind people to use the GtACH programme.

Falls specialists trained the care home staff in small, one hour group sessions, using case studies and role play to use the GtACH programme in the care homes; repeated training sessions were offered to reach all staff, including managers; and a member of care home staff was allocated to the role of falls champion, responsible for training new staff and embedding the GtACH programme. Once trained, care home staff completed the GtACH risk assessment with every resident and produced a written action plan. This initial assessment took place within four weeks of training, and reassessment was expected to take place every three to six months. The GtACH assessments guided staff to action, which staff were instructed to undertake and record in the resident's care records. Only residents recruited to the trial were followed up, and therefore the fall rate in non-recruited residents was not available for analysis.

Measures taken to guard against contamination between groups $^{12}$ comprised: explaining the importance of usual care for the control group, training staff in trial design and confidentiality agreements, collating data on staff moving to other homes in the study, and not publishing or sharing the training manual publicly. To aid recruitment, retention, and adherence to the protocol, all control homes were offered the intervention after the 12 month data had been collected and checked.

\section{Primary outcome}

The main outcome measure was the fall rate at 91-180 days after randomisation. Care home staff recorded falls in the resident's care plans and on incident forms, in keeping with usual standards of care. Every three months, researchers blinded to allocation read all the care plans and recorded the date, place, and impact of falls for all participants, including for those who had died. They cross checked the written care plans with other data held in the care home, such as incident forms, records of ambulance visits, and records of hospital admissions.

\section{Economic outcomes}

The economic evaluation measured health related quality of life using quality adjusted life years (QALYs) derived from the five domain five level version of the EuroQoL index or proxy version (EQ-5D-5L-P) ${ }^{13}$ and the Dementia Quality of Life utility measure (DEMQOL-U), ${ }^{14}$ which were completed by residents who were able to do so and by a member of care home staff as proxy (DEMQOL-P-U) for all residents until 12 months after randomisation. Baseline costs included the GtACH programme and health resource use (primary care, community health, drugs, and social service), and death were identified from care home records. Hospital use and fracture rate were obtained from routine NHS Hospital Episode Statistics reports. We applied unit costs in UK pounds sterling for 2017-18.

\section{Secondary outcomes}

Secondary outcome measures were the rate of falls at 1-90, 181-270, and 271-360 days after randomisation. Outcomes assessed at 3, 6, 9, and 12 months postrandomisation were dependency, assessed by care home staff using the Barthel index, ${ }^{15}$ a 0-20 scale with 20 indicating independence and 0 indicating a need for full care; activity, assessed using the Physical Activity Measure-Residential Care Homes (PAM-RC), ${ }^{16}$ a five item questionnaire scored out of 21 , where 21 indicates unrestricted physical activity and mobility and 0 indicates complete immobility; frequency and type of fractures at 1-6 and 7-12 months post-randomisation; and deaths anytime during 12 months post-randomisation. 
Adverse events were not recorded during the trial because the GtACH programme was considered a low risk intervention and the feasibility study had not identified specific risks, untoward incidents, or adverse events. Every month the data monitoring and ethical committees compared the fall rates between care homes to check for safety.

\section{Statistical analysis}

Supplementary material S2 provides the full statistical analysis plan. The only deviation from the initial protocol was that to better standardise reporting we relabelled the intervals for falls from months $(0-3,6-9$, and 9-12) to days (1-90, 181-270, and 271-360). The sample size was based on the primary randomised controlled trial outcome (fall rate at 91-80 days). The sample size estimate for 1474 residents recruited from 78 care homes was recalculated during the trial because of different observed mean and variability of cluster sizes from anticipated. ${ }^{9}$ The calculation assumed a fall rate of 2.5 falls each year in the control group, ${ }^{17} 80 \%$ power, a two sided significance level of $5 \%$ to detect a $33 \%$ reduction in fall rate in the intervention group (as seen in community based falls prevention interventions ${ }^{6}$ ), mean cluster size 19 , coefficient of variation 0.5 , and a $16 \%$ attrition rate.

Analyses were undertaken on an intention-to-treat basis according to a prespecified statistical analysis plan (available from author). Two sided tests were used, with statistical significance at the 5\% level. Baseline characteristics of care homes and residents and outcome measures at baseline and each followup time point were summarised by treatment arm using descriptive statistics. The fall rate was expressed as the number of falls per 1000 resident days. We compared the number of falls per resident between groups using a multilevel negative binomial regression model estimated using generalised estimating equations, with care home as the clustering variable. Primary analysis was adjusted for type of care home (residential, nursing, dual registration) and site. An additional model was fitted to assess the robustness of the model. These adjusted for fall rate for the three months before the baseline assessment. Fall rate at 1-90 days, 181-270 days, and 271-360 days were analysed as for the primary outcome. For secondary outcomes, we compared the groups using multilevel regression analysis for continuous outcomes and multilevel logistic regression for binary outcomes. In the secondary outcome analysis, we accounted for clustering by care home using a model with a random intercept for care home in all analyses. A random effect was used to account for clustering by care home. We used multilevel models in all our analyses, with the choice of model depending on the distribution of the outcome measure. Hence the class of modelling was the same for both primary and secondary outcomes, with the only difference being the specific regression model used, which was based on the type of outcome and its distribution. All analyses were prespecified and carried out using STATA 16.1 .
Quality of life scores were converted to utilities, ${ }^{18} 19$ from which quality adjusted life years (QALYs) were calculated using linear interpolation and area under the curve analysis with baseline adjustment. If residents died, their utility value and costs were assumed to be zero from the subsequent assessment point and were retained. Costs and outcomes were not discounted, reflecting the timeframe for the analysis. Mean cost and outcomes data were combined to calculate incremental cost effectiveness ratios for both QALY measures, adjusted for age, sex, and site. The cost per fall averted was calculated. Analyses of costs and outcomes used generalised estimating equations regression models. In sensitivity analyses we performed multiple imputation using chained equations using the mi impute command in STATA version $16 .^{20}$ The multiple imputation model included predictors of secondary and non-secondary care costs (baseline and full follow-up); EQ5D-5L and DEMQOL-P based QALYs; and treatment group, care home, age, and sex. The imputation generated values for missing data at each follow-up using ordinary least squares, generating 50 datasets. The generalised estimating equations models were then run on each of these, and the outputs pooled using Rubin's rules. ${ }^{21}$ This enabled paired cost and outcome data for the entire study population. This was repeated 200 times, with bootstrap replications of the original data. Paired bootstrapped estimates of incremental cost and utility were generated to produce a scatterplot of incremental cost-outcome pairs and the cost effectiveness acceptability curve. All regression analysis was conducted in STATA MP $16^{22}$ and $\mathrm{R}^{23}$

\section{Patient and public involvement}

A patient and public involvement (PPI) team were instrumental in securing funding, influencing the trial set up, and advocating for care home residents throughout the trial. An adaptation of the research cycle was examined to plan for PPI involvement at each stage of the study, as advocated by UK Standards for Public Involvement. ${ }^{24}$ The aim was to ensure the trial had relevance to care home residents, parties interested in care homes, and the public. The GRIPP2 short form framework $^{25}$ was used to ensure consistency. This approach captures the unique perspective of patients and public experience at each stage of the research cycle. All public facing documents were reviewed by PPI representatives, and PPI members participated as lay researchers in the process analysis. PPI representatives attended all project management meetings. PPI representatives are already working with the research team on a follow-up implementation study, where they are helping us co-design, in conjunction with care home staff representatives, a package to implement the findings from the work presented in this paper. A PPI representative is a co-author on this paper.

\section{Results}

Recruitment took place between 1 November 2016 and 31 January 2018. Eighty four care homes were randomised: 39 to the GtACH programme and 45 
to usual care. This imbalance was related to the stratification by site and type and uneven recruitment of homes by type across sites. Overall, 1657 residents consented and provided baseline measures. Consent was obtained for an average of $50 \%$ of residents from participating care homes, and an average of 19.5 participants resided in each care home. GtACH training was delivered to 1051/1480 staff in 146 group sessions, representing $71 \%$ of care home staff. Figure 1 shows the flow of care homes and residents through the study. Table 1 shows the characteristics of the care homes and residents.

\section{Outcomes}

Data for the primary outcome, fall rate at 91-180 days after randomisation, were available for 630 residents assigned to GtACH and 712 assigned to usual care (table 2). The fall rate over this period was $6.0 / 1000$ resident days in the GtACH group and 10.4/1000 resident days in the usual care group. The unadjusted incidence rate ratio of 0.57 (95\% confidence interval 0.45 to $0.71, \mathrm{P}<0.001$ ) favoured $\mathrm{GtACH}$; after adjusting for baseline falls rate, the incidence rate ratio was similar (0.63, 0.52 to $0.78, \mathrm{P}<0.001)$. A significantly lower fall rate was found in the GtACH group at 1-90 days after randomisation, but not at $181-270$ or $271-360$ days after randomisation (table 2). No differences were found between groups in any of the other secondary outcomes; other than a lower proportion of residents sustaining fractures in the GtACH group at 181-360 days after randomisation (table 3 and table 4).

\section{Health economic outcomes}

The final dataset for the economic analysis comprised 1603 residents (732 in the GtACH group and 871 in the usual care group). Completion rates for data were high, with no more than 283/1603 (17.7\%) items missing from any individual variable (DEMQOL based QALYs) and complete datasets available for $1260 / 1603$ $(78.6 \%)$ residents. Six of the 1603 residents $(0.4 \%)$ were missing cost data, $13(0.8 \%)$ and $15(0.9 \%)$ were missing baseline EQ5D and DEMQOL utility data, and $262(16.3 \%)$ and $283(17.7 \%)$ were missing EQ-5D and DEMQOL based QALYs, respectively.

Table 5 shows the incremental costs per resident for the GtACH programme and the difference in outcomes between the groups (QALYs and falls over 12 months). Table 6 shows the incremental cost effectiveness ratios. The primary analysis showed the incremental cost per EQ-5D-5L-P based QALY to be $€ 4544$ and per DEMQOLP-U based QALY to be $£ 20889$. The cost per fall averted was $£ 191$. The results of the sensitivity analysis were similar to those of the base case. The cost effectiveness acceptability curve showed a $92 \%$ probability that the GtACH programme was cost effective at a £20000 per QALY threshold using QALYs based on the EQ-5D5L-P, and a 57\% probability using QALYs based on the DEMQOL-P-U. Supplementary table S3 shows the costs to deliver the GtACH programme per resident. Supplementary table S4 shows the incremental cost for each individual cost component. Supplementary table
S5 shows mean utility scores by follow-up period and QALYs for DEMQOL-P-U and EQ-5D-5L at 12 months. Supplementary figures S6-S8 show scatterplots and cost effectiveness acceptability curves for EQ-5D-5L-P based QALYs, DEMQOL based QALYs, and the cost per fall averted, respectively.

\section{Discussion}

The Guide to Action for Care Homes (GtACH) programme was associated with a statistically significant reduction in fall rate in care home residents in the period 3-6 months after randomisation, which was the primary outcome of this study. The reduction in falls was achieved with no effect on death, dependency, or activity.

The GtACH programme was within conventional thresholds of cost effectiveness when health related quality of life was estimated using the EQ-5D-5L-P.

Considering secondary outcomes, these aligned with the primary outcome, showing a significant reduction in falls at months 1-3 after randomisation. There was also an indication that use of the GtACH could be associated with fewer fractures-this has potentially important cost saving implications for health services.

\section{Strengths and weaknesses of this study}

The strengths of this study relate to the large and representative sample size, the measures taken to avoid contamination, and the comprehensive approach to costing and health economics. Few data were missing, partly because data were collected quarterly. We consider that training $71 \%$ of care home staff was a success, given that such staff often work part time or out of office hours, and given the extent of staff turnover in the care home sector.

A weakness of the study was that we were unable (owing to ethical approvals) to collect falls data from residents not in our study who may also have been exposed to the intervention, or not, depending on the arm to which they were randomised. Our recruitment process might have selected care homes with staff who had a particular interest in falls prevention, meaning that usual care in our control homes might have been better than usual care seen more widelythis might have been expected to reduce the size of the treatment effect and is unlikely to negate our findings. It is possible that the intervention could have influenced the reporting of falls differentially between intervention and control arms because of the nature of GtACH. Although it is difficult to be sure about the directionality of such a difference, the focus on reporting falls to trigger the GtACH intervention could well have increased falls reporting in intervention homes compared with control homes. Thus it is unlikely that such a difference would have contributed to an overestimation of treatment effect. The loss of the treatment effect after six months could be due to a loss of statistical power owing to high attrition from death, but even if this was a genuine loss of effect, the short life expectancy of care home residents might make a short lived treatment effect acceptable. Another 
(186 186

Care homes assessed for eligibility

Care homes excluded
55 Did not meet inclusion criteria*
25 Recruitment closed before consent
19 Other reasonst

\section{7}

Care homes recruited

\section{( 1698}

Residents recruited and baseline

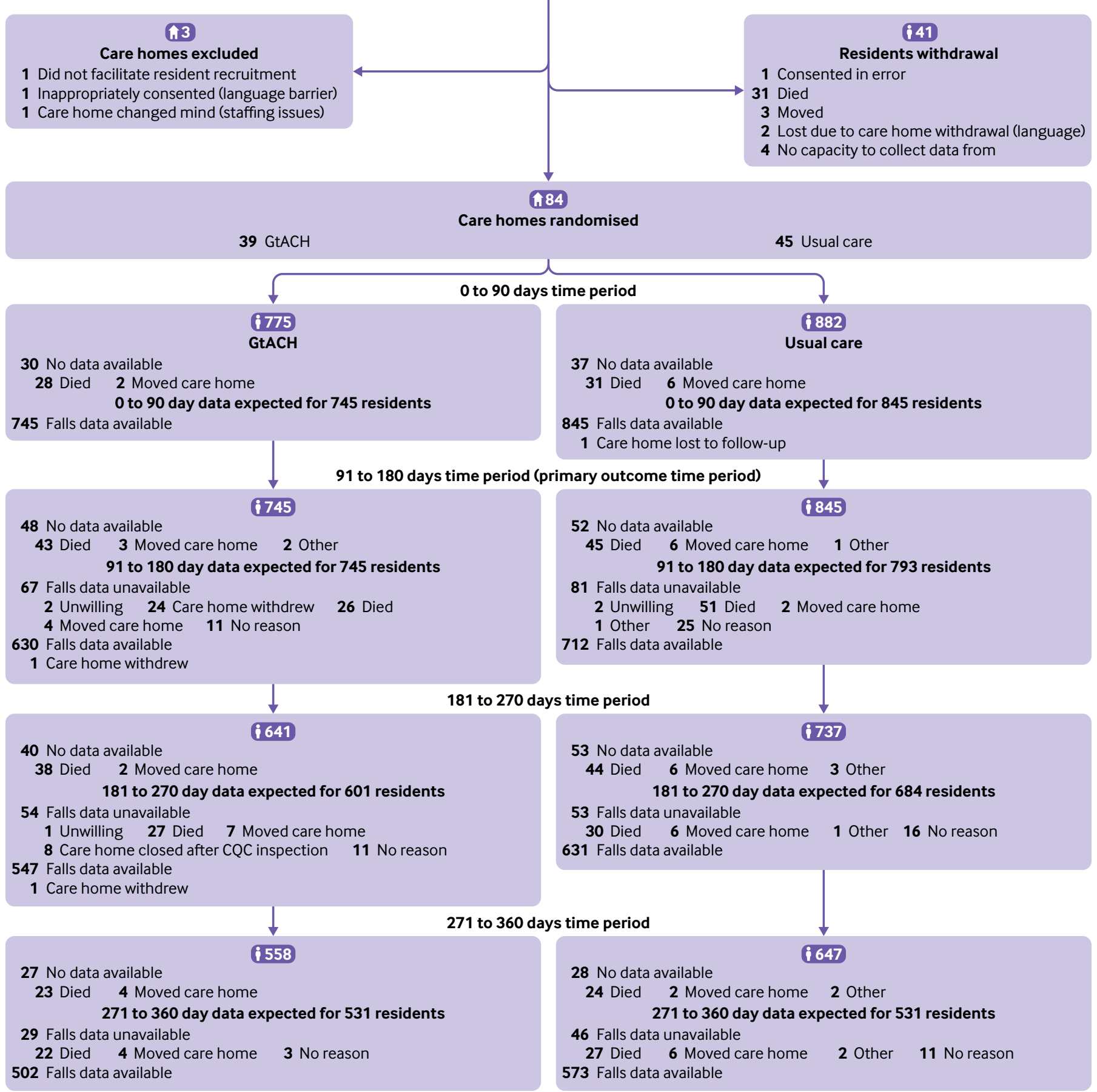

Fig 1 | Flow of and care homes and residents through study. *Not prepared to allocate a falls champion ( $n=24)$, existing falls programme ( $n=13)$, participated in previous studies $(n=4)$, learning disability $(n=3)$, under review $(n=1)$, no reason given $(n=10)$. tDid not have time $(n=1)$, stopped communicating with researcher $(n=15)$, adopted a falls intervention $(n=3)$. Care homes considered lost to follow-up did not respond to multiple requests for follow-up data collection. GtACH=Guide to Action for Care Homes programme; $C Q C=$ Care Quality Commission 


\begin{tabular}{|c|c|c|c|}
\hline Characteristics & Overall $(n=84)$ & GtACH group $(n=39)$ & Usual care group $(n=45)$ \\
\hline \multicolumn{4}{|l|}{ Care homes } \\
\hline \multicolumn{4}{|l|}{ Type of care home: } \\
\hline Nursing & $11(13)$ & $5(13)$ & $6(13)$ \\
\hline Residential & $34(40)$ & $16(41)$ & $18(40)$ \\
\hline Dual registration & $39(46)$ & $18(46)$ & $21(47)$ \\
\hline Total No of care giving staff & 3609 & 1491 & 2118 \\
\hline Median (IQR) No of care giving staff per home & $33(25-50)$ & $38(24-50)$ & $47(25-48)$ \\
\hline Total No of beds & 4112 & 1912 & 2200 \\
\hline Median (IQR) No of beds per home & $41(33-62)$ & $43(33-64)$ & $41(33-60)$ \\
\hline Total No of residents & 3561 & 1672 & 1889 \\
\hline Median (IQR) No of residents per home & $36(27-53)$ & $39(28-59)$ & $34(26-48)$ \\
\hline Median (IQR) No of recruited residents per home & $18(15-22)$ & $18(12-22)$ & $18(15-22)$ \\
\hline \multicolumn{4}{|l|}{ Residents } \\
\hline Total No & 1657 & 775 & 882 \\
\hline Mean (SD) age (years) & $85.0(9.3)$ & $86.0(8.6)$ & $84.2(9.7)$ \\
\hline Men & $532(32.1)$ & $231(29.8)$ & $301(34.1)$ \\
\hline Consent: resident & $387(23.4)$ & $186(24.0)$ & $201(22.8)$ \\
\hline Consultee & $1270(76.6)$ & $589(76.0)$ & $681(77.2)$ \\
\hline Median (IQR) months in care home & $18.6(8.3-36.4)$ & $18.8(8.1-36.5)$ & $18.1(8.6-35.8)$ \\
\hline Dementia & $1109(67.0)$ & $506(65.4)$ & $603(68.4)$ \\
\hline Diabetes & $320(19.3)$ & $150(19.4)$ & $170(19.3)$ \\
\hline Stroke & $262(15.8)$ & $118(15.2)$ & $144(16.3)$ \\
\hline Coronary heart disease & $234(14.1)$ & $100(12.9)$ & $134(15.2)$ \\
\hline Mean (SD) falls/person in 3 months before baseline & $0.71(1.82)$ & $0.61(1.57)$ & $0.79(2.02)$ \\
\hline Mean (SD) PAM-RC score & $8.61(6.09)$ & $8.57(5.95)$ & $8.66(6.21)$ \\
\hline Mean (SD) Barthel index score & $8.57(6.05)$ & $8.86(6.12)$ & $8.30(5.99)$ \\
\hline EQ-5D-5L self-completion & $0.49(0.36)$ & $0.52(0.36)$ & $0.46(0.35)$ \\
\hline EQ-5D-5L proxy & $0.35(0.37)$ & $0.36(0.37)$ & $0.34(0.36)$ \\
\hline DEMQOL-U self-completion & $0.82(0.16)$ & $0.83(0.16)$ & $0.81(0.16)$ \\
\hline DEMQOL-U proxy & $0.74(0.12)$ & $0.74(0.12)$ & $0.74(0.12)$ \\
\hline
\end{tabular}

*Care home managers or next of kin who helped to recruit residents.

PAM-RC=Physical Activity Measure-Residential Care Homes; EQ-5D-5L=five domain five level version of EuroQoL index (Crosswalk ${ }^{18}$ value set ranges from utility of 1 for best imaginable health to

-0.59 for worst imaginable health); DEMQOL-U=Dementia Quality of Life utility measure.

explanation for the loss of treatment effect after six months could be a waning of falls awareness, and it is possible that further support or training at this time could have perpetuated the treatment effect. This study was not powered to detect a difference in fractures between groups. We consider that the significantly lower proportion of participants in the GtACH group who sustained one or more fractures in the period 181360 days after randomisation is interesting, but the analysis was based on small numbers and there was no corresponding reduction in falls over that period.

The number of care homes between groups showed a slight imbalance (39 in the GtACH arm and 45 in the control arm) because we had a limited number of care homes with some of the characteristics required for stratified randomisation. Despite this, the randomisation list still had a 1:1 ratio in each stratum and we therefore do not think that this influenced our findings. The median number of staff per home differed between the control $(n=47)$ and GtACH $(n=38)$ arms, despite a similar median number of beds. This means that the staffing ratios were lower in the intervention homes. Staffing of care homes is complex and is influenced by factors such as funding models; the geographical location of care homes, which impacts on availability of staff; and the ethos and culture of the provider organisation. ${ }^{26}$ Staffing ratios in care homes are not closely related to resident dependency in the UK as they are in other countries. We did not find a substantive difference in other baseline variables that

\begin{tabular}{|c|c|c|c|c|c|c|c|c|c|c|}
\hline \multirow[b]{2}{*}{$\begin{array}{l}\text { Fall rate } \\
\text { outcome }\end{array}$} & \multicolumn{3}{|c|}{ GtACH group } & \multicolumn{3}{|c|}{ Usual care group } & \multirow[b]{2}{*}{$\begin{array}{l}\text { Minimally adjusted* } \\
\text { incidence rate ratio } \\
(95 \% \mathrm{Cl})\end{array}$} & \multirow[b]{2}{*}{$P$ value } & \multirow[b]{2}{*}{$\begin{array}{l}\text { Fully adjustedt } \\
\text { incidence rate ratio } \\
(95 \% \mathrm{Cl})\end{array}$} & \multirow[b]{2}{*}{$P$ value } \\
\hline & $\begin{array}{l}\text { No at } \\
\text { risk }\end{array}$ & $\begin{array}{l}\text { Mean (SD) falls } \\
\text { per participant }\end{array}$ & $\begin{array}{l}\text { Mean (SD) fall } \\
\text { rate per } 1000 \\
\text { resident days }\end{array}$ & $\begin{array}{l}\text { No at } \\
\text { risk }\end{array}$ & $\begin{array}{l}\text { Mean (SD) falls } \\
\text { per participant }\end{array}$ & $\begin{array}{l}\text { Mean (SD) fall } \\
\text { rate per } 1000 \\
\text { resident days }\end{array}$ & & & & \\
\hline \multicolumn{11}{|c|}{ Primary outcome } \\
\hline 91-180 days & 630 & $0.49(1.13)$ & $6.04(14.02)$ & 712 & $0.89(2.60)$ & $10.38(29.52)$ & $0.57(0.45$ to 0.71$)$ & $<0.001$ & $0.63(0.52$ to 0.78$)$ & $<0.001$ \\
\hline \multicolumn{11}{|c|}{ Secondary outcomes } \\
\hline 1-90 days & 708 & $0.55(1.36)$ & $6.93(20.56)$ & 826 & $0.88(2.37)$ & $10.24(27.26)$ & $0.6(0.49$ to 0.73$)$ & $<0.001$ & 0.74 (0.60 to 0.92) & 0.006 \\
\hline 181-270 days & 547 & $0.60(1.29)$ & $7.28(16.67)$ & 633 & $0.73(1.85)$ & $9.21(28.77)$ & 0.85 (0.69 to 1.05$)$ & 0.13 & 0.91 (0.74 to 1.12$)$ & 0.37 \\
\hline $271-360$ days & 502 & $0.55(1.14)$ & $6.22(12.88)$ & 573 & $0.79(2.37)$ & $9.22(27.36)$ & $0.79(0.60$, to 1.03$)$ & 0.08 & 0.93 (0.71 to 1.22$)$ & 0.61 \\
\hline
\end{tabular}




\begin{tabular}{|c|c|c|c|c|c|c|c|c|}
\hline \multirow{2}{*}{ Days } & \multicolumn{2}{|c|}{ GtACH group } & \multicolumn{2}{|c|}{ Usual care group } & \multirow{2}{*}{$\begin{array}{l}\text { Minimally adjusted* mean } \\
\text { difference }(95 \% \mathrm{Cl})\end{array}$} & \multirow[b]{2}{*}{$P$ value } & \multirow{2}{*}{$\begin{array}{l}\text { Fully adjustedt mean } \\
\text { difference }(95 \% \mathrm{CI})\end{array}$} & \multirow[b]{2}{*}{$P$ value } \\
\hline & No & Mean (SD) & No & Mean (SD) & & & & \\
\hline \multicolumn{9}{|c|}{ Barthel index score: } \\
\hline 90 & 643 & $8.24(6.12)$ & 726 & $7.87(5.94)$ & $0.08(-0.96$ to 1.13$)$ & 0.87 & $-0.03(-0.69$ to 0.64$)$ & 0.94 \\
\hline 180 & 584 & $8.12(6.05)$ & 648 & $7.54(5.86)$ & $0.16(-0.89$ to 1.20$)$ & 0.77 & $-0.02(-0.48$ to 0.43$)$ & 0.93 \\
\hline 270 & 514 & $8.52(6.17)$ & 576 & $7.18(5.98)$ & $0.90(-0.29$ to 2.10$)$ & 0.14 & $0.46(-0.10$ to 1.01$)$ & 0.11 \\
\hline 360 & 447 & $8.11(6.20)$ & 519 & $6.86(5.92)$ & $0.82(-0.32$ to 1.96$)$ & 0.16 & $0.44(-0.26$ to 1.15$)$ & 0.21 \\
\hline \multicolumn{9}{|c|}{ PAM-RC scores: } \\
\hline 90 & 652 & $7.99(6.01)$ & 736 & $8.16(5.98)$ & $-0.41(-1.51$ to 0.69$)$ & 0.47 & $-0.1(-0.55$ to 0.35$)$ & 0.66 \\
\hline 180 & 578 & $8.11(6.05)$ & 633 & $7.74(6.08)$ & $0.07(-1.04$ to 1.17$)$ & 0.91 & $0.23(-0.28$ to 0.75$)$ & 0.38 \\
\hline 270 & 491 & $8.13(5.98)$ & 576 & $7.59(6.12)$ & $0.32(-0.90$ to 1.54$)$ & 0.61 & $0.43(-0.24$ to 1.10$)$ & 0.21 \\
\hline 360 & 439 & $7.96(5.63)$ & 520 & $7.19(6.03)$ & $0.45(-0.57$ to 1.47$)$ & 0.39 & $0.49(-0.16$ to 1.14$)$ & 0.14 \\
\hline
\end{tabular}

would indicate these staffing ratios reflect differing resident dependency across the intervention and control arms.

A limitation was the number of homes that could not participate because they did not agree to appoint a falls champion or stopped communicating with the researcher. Thus, the study might have selected care homes that were better led, organised, or resourced. It is well recognised that care homes that support research are by definition of volunteering to participate in research, always atypical. ${ }^{27}$ We can, however, be confident that the age, functional dependency, sex, and comorbidities of the residents were similar to those of other care home research ${ }^{3}$ and that our protocol enabled us to sample from a wide range of organisations for size, registration status, and specialisation. These findings are likely to be as generalisable as any care home research. Our next project is to consider how we can implement these findings across the wider range of care homes that do not routinely participate in research studies.

\section{Strengths and weaknesses in relation to other studies}

Cochrane systematic reviews have shown that the fall rate in older people living in their own home can be reduced, but research presented in the 2018 Cochrane review of 13 care facilities were inconclusive about multifactorial interventions for falls prevention. ${ }^{6}$ The studies were classified as very low or low quality. Our study provides different findings.

An important question is why this study might have been successful in showing a reduction in falls, whereas others have not found such outcomes. An important difference between this study and previously published work relates to the earlier processes of codesign, piloting, and feasibility trials, ${ }^{78}$ which enabled us to design an intervention that was sensitive in the context of care homes and cognisant of the specific challenges of falls prevention in this sector. This is in keeping with an increasing body of evidence, which found that interventions that assess and take account of the care home context, ${ }^{28}$ and which empower care home staff and organisations as partners in design and implementation, ${ }^{29}$ are more likely to be successful.

A limitation of our study common to falls studies was that although the outcomes were collected by researchers blinded to allocation, participants and care home staff were not blinded and they recorded the falls in care records. The direction of any bias resulting from this is unclear and it is likely that any bias would be small because UK care homes have a statutory duty of care to record falls. We checked the falls recording processes at each follow-up, and all care homes used the same falls definition. We consider that our interest in falls might have increased the accuracy of falls recording in the care homes, as the care home staff recognised they were participating in a study.

\begin{tabular}{|c|c|c|c|c|c|c|c|c|}
\hline \multirow{2}{*}{$\begin{array}{l}\text { Events by follow-up } \\
\text { (days) }\end{array}$} & \multicolumn{2}{|c|}{ GtACH group } & \multicolumn{2}{|c|}{ Usual care group } & \multirow[b]{2}{*}{$\begin{array}{l}\text { Minimally adjusted* } \\
\text { odds ratio }(95 \% \mathrm{Cl})\end{array}$} & \multirow[b]{2}{*}{$P$ value } & \multirow[b]{2}{*}{$\begin{array}{l}\text { Fully adjustedt odds } \\
\text { ratio }(95 \% \mathrm{Cl})\end{array}$} & \multirow[b]{2}{*}{ Pvalue } \\
\hline & No & $\begin{array}{l}\text { No (\%) with adverse } \\
\text { outcome }\end{array}$ & No & $\begin{array}{l}\text { No (\%) with adverse } \\
\text { outcome }\end{array}$ & & & & \\
\hline \multicolumn{9}{|l|}{ Fractures ( $\geq 1)$ : } \\
\hline $0-180$ & 775 & $33(4.3)$ & 822 & $42(4.8)$ & 1.19 (0.70 to 2.01$)$ & 0.53 & - & - \\
\hline $181-360$ & 600 & $9(1.5)$ & 685 & $26(3.8)$ & $0.34(0.15$ to 0.75$)$ & 0.007 & 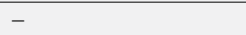 & - \\
\hline \multicolumn{9}{|l|}{ Deaths: } \\
\hline $1-360$ & 775 & $233(30.1)$ & 882 & 281 (31.9) & $0.93(0.73$ to 1.20$)$ & 0.58 & - & - \\
\hline \multicolumn{9}{|l|}{ Fallers $((\geq 1)$ : } \\
\hline $1-90$ & 708 & $194(27.4)$ & 826 & $266(32.2)$ & $0.70(0.50$ to 1.00$)$ & 0.05 & 0.75 (0.53 to 1.05$)$ & 0.09 \\
\hline $91-180$ & 630 & $167(26.5)$ & 712 & $216(30.3)$ & $0.76(0.56$ to 1.03$)$ & 0.08 & 0.81 (0.60 to 1.10$)$ & 0.18 \\
\hline $181-270$ & 547 & $165(30.2)$ & 633 & $187(29.5)$ & $1.00(0.73$ to 1.37$)$ & 0.99 & $1.06(0.78$ to 1.45$)$ & 0.70 \\
\hline $271-360$ & 502 & $147(29.3)$ & 573 & $175(30.5)$ & 0.88 (0.60 to 1.29 ) & 0.52 & 0.94 (0.65 to 1.37$)$ & 0.75 \\
\hline
\end{tabular}




\begin{tabular}{|c|c|c|c|c|c|c|}
\hline & \multicolumn{2}{|c|}{ GtACH group } & \multicolumn{2}{|c|}{ Usual care group } & \multicolumn{2}{|l|}{ Incremental mean $(95 \% \mathrm{Cl})$} \\
\hline & No & Mean (SD) cost (f) & No & Mean (SD) cost (f) & Primary analysis & Multiple imputation analysis \\
\hline \multicolumn{7}{|l|}{ Costs } \\
\hline Base case $^{\star}$ & 732 & $3955.29(3949.38)$ & 865 & $3935.54(3879.9)$ & $108.26(-271.06$ to 487.58$)$ & $108.26(-232.89$ to 449.41$)$ \\
\hline \multicolumn{7}{|l|}{ Sensitivity analyses } \\
\hline Repeat GtACH uset & 732 & $3978.2(3955.87)$ & 865 & $3935.54(3879.9)$ & $131.81(-247.77$ to 511.4$)$ & $131.81(-209.28$ to 472.9$)$ \\
\hline Extra mortality costsł & 732 & $4103.96(4121.02)$ & 865 & $4047.89(3989.66)$ & $124.98(-268.68$ to 518.64$)$ & $124.98(-230.84$ to 480.8$)$ \\
\hline $\begin{array}{l}\text { Repeat GtACH use and extra } \\
\text { mortality costs§ }\end{array}$ & 732 & $4126.87(4127.1)$ & 865 & $4047.89(3989.66)$ & $148.52(-245.4$ to 542.45$)$ & $148.52(-207.33$ to 504.38$)$ \\
\hline \multicolumn{7}{|l|}{ Outcomes } \\
\hline EQ-5D-5L Proxy based QALYS & 622 & $0.266(0.317)$ & 718 & $0.232(0.291)$ & 0.024 (0.004 to 0.044)ף & 0.023 (0.003 to 0.043$) 9$ \\
\hline DEMQOL-P-U based QALYS & 611 & $0.578(0.24)$ & 708 & $0.581(0.235)$ & $0.005(-0.019$ to 0.03$) \rrbracket$ & $0.005(-0.018$ to 0.029$)$ q \\
\hline Falls over 12 months & 732 & $1.889(3.662)$ & 871 & $2.747(7.414)$ & $-0.568(-0.97$ to -0.166$)$ ף & $-0.574(-0.961$ to -0.186$) \uparrow$ \\
\hline \multicolumn{7}{|c|}{$\begin{array}{l}£ 1.00 \text { ( } € 1.19 ; \$ 1.35) \text {. } \\
\text { EQ-5D-5L=five domain five level version of the EuroQoL index (Crosswalk }{ }^{18} \text { value set ranges from utility of } 1 \text { for best imaginable health to }-0.59 \text { for worst imaginab } \\
\mathrm{U}=\text { Dementia Quality of Life utility measure (utility ranges from } 0.363 \text { to } 0.937 \text { for DEMQOL-U-P (proxy) and } 0.986 \text { to } 0.243 \text { on DEMQOL-U, both value sets derived } \\
\text { *Based on intervention costs assuming GtACH tool is used once per resident as this was reflective of what the process evaluation team observed. The base case an } \\
\text { care costs related to end of life care and mortality. } \\
\text { †This analysis costs the intervention assuming the maximum number of repeat GtACH's each care home could provide would be one per month if the resident fell. } \\
\text { fThis analysis incorporates secondary care costs related to mortality. } \\
\text { §This sensitivity analysis incorporates both repeated GtACH use and mortality costs. } \\
\text { १Mean difference ( } 95 \% \text { confidence interval). }\end{array}$} \\
\hline
\end{tabular}

Although GtACH was found to be cost effective using the EQ-5D-5L, it was of borderline cost effectiveness when the DEMQOL-P-U was used. Research published after our study had started indicates that the EQ-5D performs better than the DEMQOL in care homes. ${ }^{21} 30$ EQ-5D has been shown to be more responsive to change, ${ }^{31}$ which is the justification for our conclusion that the GtACH programme was cost effective. The DEMQOL focuses more on the emotional impact of dementia, whereas in this study the outcome was not dementia specific, and not all residents had dementia. The mean utility for self-reported quality of life indices was higher than that for proxy report. This might reflect that those with earlier dementia, and hence able to complete self-report questionnaires, had better quality of life. It might also be a consequence of the limited agreement between proxy and self-report quality of life indices when used in the care home population, ${ }^{32}$ which is well described as a limitation that affects all studies conducted in care homes and is an area of ongoing research.

\section{Policy implications}

This study provides findings that confirm the hypothesis that an intervention which includes all of awareness raising, education, screening, decision support, and implementation support can reduce falls in care homes. It is possible that the intervention succeeded because of its comprehensiveness and as a result of the recognition it gave to the pivotal role played by care home staff in designing, implementing, and delivering the GtACH programme in this setting. ${ }^{33}$ Clinicians and policy makers should, when working to prevent falls in care homes, implement interventions that are similarly comprehensive in scope and that include each of the components included in GtACH. There are always caveats associated with extrapolating such findings to care homes that might differ

\begin{tabular}{|c|c|c|}
\hline Incremental cost effectiveness ratios & Primary analysis (f) & Multiple imputation analysis (f) \\
\hline \multicolumn{3}{|l|}{ Base case } \\
\hline Per EQ-5D-5L-P based QALY & 4543.69 & 4651.63 \\
\hline Per DEMQOL-P-U based QALY & 20889.42 & 20557.80 \\
\hline \multicolumn{3}{|l|}{ Sensitivity analyses } \\
\hline \multicolumn{3}{|l|}{ With repeat GtACH use: } \\
\hline Per EQ-5D-5L-P based QALY & 5532.14 & 5663.56 \\
\hline Per DEMQOL-P-U based QALY & 25433.80 & 25030.04 \\
\hline \multicolumn{3}{|l|}{ With extra mortality cost: } \\
\hline Per EQ-5D-5L-P based QALY & 5245.37 & 5369.98 \\
\hline Per DEMQOL-P-U based QALY & 24115.39 & 23732.56 \\
\hline \multicolumn{3}{|l|}{ With repeat GtACH and extra mortality cost: } \\
\hline Per EQ-5D-5L-P based QALY & 6233.50 & 6381.58 \\
\hline Per DEMQOL-P-U based QALY & 28658.26 & 28203.32 \\
\hline \multicolumn{3}{|l|}{ Incremental cost per fall averted } \\
\hline Base case & 190.62 & 188.72 \\
\hline With repeat GtACH use & 232.09 & 229.77 \\
\hline With extra mortality cost & 220.06 & 217.86 \\
\hline With repeat GtACH use and extra mortality cost & 261.52 & 258.91 \\
\hline
\end{tabular}


structurally, culturally, or organisationally from those included in the study, but we do not believe that these issues of generalisability can be addressed by further trials. Rather, it is implementation research that is required to explain how to realise these benefits across a wider range of settings.

This was a complex intervention, and key to understanding the implications for service delivery is that a process evaluation was completed concurrently to this trial and provided insights into how care homes received and delivered $\mathrm{GtACH}$, including how behaviours and processes changed in relation to the programme. We have captured these data-both quantitative and qualitative-in the process evaluation and plan to publish the results separately to enable all relevant detail to be shared.

Although this work was conducted in the UK, where care homes are run by private providers and not managed by the NHS, these findings are likely to be generalisable to other long term care settings for older people internationally, where the incidence of falls is high and where nurses and care assistants without specialist training in falls prevention predominantly provide day-to-day delivery of care. ${ }^{34}$ In the same way that further research is required and planned, to understand the generalisability of our findings to other non-participant UK care homes, similar work is required to explore how to accommodate our findings in long term sectors internationally where conditions might differ from those in the UK.

\section{Unanswered questions and future research}

Future work should aim to implement the GtACH programme in care homes where a systematic and equivalent fall prevention programme is not in place. Implementation in care homes is not straightforward and must take account of substantial variation in ethos and organisational structure between care home operators. ${ }^{28}$ It will be important, therefore, to research how to implement GtACH consistently and sustainably across different health and social care systems, and at scale. We are currently undertaking further research to understand how to consistently implement the GtACH programme in care homes. The research will also develop digital materials and online training to support this.

\section{Conclusion}

Our multifactorial falls prevention intervention in UK care homes was associated with a reduction in fall rate and cost effectiveness, without a reduction in activity or increase in dependency. Further research working closely with the care home sector is required to understand how to implement this type of intervention consistently across the full range of care home provider organisations.

AUTHOR AFFILIATIONS

${ }^{1}$ School of Medicine, Queens Medical Centre, University of

Nottingham, Nottingham, NG7 2UH, UK

${ }^{2}$ Nottingham CityCare Partnership, Nottingham, UK
${ }^{3}$ NIHR Applied Research Collaboration - East Midlands, Nottingham, UK

${ }^{4} \mathrm{NIHR}$ Nottingham Biomedical Research Centre, Nottingham, UK ${ }^{5}$ Nottingham University Hospitals NHS Trust, Nottingham, UK ${ }^{6}$ University Hospitals of Derby and Burton NHS Foundation Trust, Derby, UK

${ }^{7}$ Norwich Medical School, University of East Anglia, Norwich, UK

${ }^{8}$ University of Hertfordshire, Hatfield, UK

${ }^{9}$ Exeter Medical School, University of Exeter, Exeter, UK

${ }^{10}$ University College London, London, UK

${ }^{11}$ University of Bradford, Bradford, UK

${ }^{12}$ Nottingham, UK

We thank the nursing home managers, staff, residents, and informal carers who generously gave their time and participated in the study.

Contributors: PAL (chief investigator) designed and led trial delivery and wrote the paper. She is the guarantor. JCH and ES led trial delivery and contributed intellectual content to the paper. FA, JD, KM, and KR collected and analysed data and wrote the paper. SA, AC, and SS did the statistical analysis, and accessed and verified the data. TS, ECFW, and $\mathrm{LI}$ did the health economic analysis. SC, CF, GM, ALG, JRFG, and $\mathrm{KR}$ led data collection, and contributed to data analysis and writing the paper. MG was the patient and public involvement representative and contributed to all aspects of the study. The corresponding author attests that all listed authors meet authorship criteria and that no others meeting the criteria have been omitted.

Funding: This study was funded by the National Institute for Health Research (NIHR) HTA programme (ref 13/115/29). PAL, JCH, JRFG, and ALG are funded in part by the NIHR Applied Research CollaborationEast Midlands (ARC-EM). PAL, JRFG, and ALG are funded in part by the NIHR Nottingham Biomedical Research Centre. The views expressed are those of the authors and not necessarily those of the NIHR, NHS, or Department of Health and Social Care.

Competing interests: All authors have completed the ICMJE uniform disclosure form at www.icmje.org/disclosure-of-interest/ and declare: funding from the National Institute for Health Research; no support from any organisation for the submitted work; no financial relationships with any organisations that might have an interest in the submitted work in the previous three years; no other relationships or activities that could appear to have influenced the submitted work.

Ethical approval: This study was approved by the Yorkshire and Humber-Bradford Leeds research ethics committee (REC 16/ $\mathrm{YH} / 0111)$.

Data sharing: Data requests should be submitted to the corresponding author for consideration. Data collected for the study, including anonymised individual participant data and a data dictionary defining each field in the set, will be made available to others after review.

The lead author (PAL) affirms that the manuscript is an honest, accurate, and transparent account of the study being reported; that no important aspects of the study have been omitted; and that any discrepancies from the study as planned (and, if relevant, registered) have been explained

Dissemination to participants and related patient and public communities: We have presented results at national and international conferences and in addition have been awarded an implementation grant from the National Institute for Health Research Applied Research Collaboration to investigate how to improve uptake. We anticipate that further, lay facing, publications and resources will be developed as part of this further work. The findings will also be incorporated into national guidelines.

Provenance and peer review: Not commissioned; externally peer reviewed.

This is an Open Access article distributed in accordance with the terms of the Creative Commons Attribution (CC BY 4.0) license, which permits others to distribute, remix, adapt and build upon this work, for commercial use, provided the original work is properly cited. See: http://creativecommons.org/licenses/by/4.0/.

1 Public Health England. Falls: Applying All Our Health. www.gov.uk/ government/publications/falls-applying-all-our-health/falls-applyingall-our-health

2 Laing W. Care homes for Older People market analysis and projections. 2017. www.laingbuissonevents.com/wp-content/ uploads/2017/05/William-COP.pdf 
3 Gordon AL, Franklin M, Bradshaw L, Logan P, Elliott R, Gladman JR. Health status of UK care home residents: a cohort study. Age Ageing 2014:43:97-103. doi: 10.1093/ageing/aft077

4 Inspectorate. TC. Managing Falls and Fractures in Care Homes for Older People. 2016. www.careinspectorate.com/images/ documents/2737/2016/Falls-and-fractures-new-resource-low-res.pdf

5 Oliver D. Preventing falls and falls-injuries in hospitals and long-term care facilities. Rev Clin Gerontol 2007;17:75-91. doi: 10.1017/ S0959259808002451

6 Cameron ID, Dyer SM, Panagoda CE, et al. Interventions for preventing falls in older people in care facilities and hospitals. Cochrane Database Syst Rev 2018;9:CD005465. . doi: 10.1002/14651858.CD005465.pub4

7 Robertson K, Logan P, Ward M, et al. Thinking falls-taking action: a falls prevention tool for care homes. $\mathrm{Br}$ J Community Nurs 2012;17:206-9. doi: 10.12968/bjcn.2012.17.5.206

8 Walker GM, Armstrong S, Gordon AL, et al. The Falls In Care Home study: a feasibility randomized controlled trial of the use of a risk assessment and decision support tool to prevent falls in care homes. Clin Rehabil 2016;30:972-83. doi: 10.1177/0269215515604672

9 Logan P, McCartney K, Armstrong S, et al. Evaluation of the Guide to Action Care Home fall prevention progamme in care homes for older people: protocol for a multi-centre, single blinded, cluster randomised controlled trial (FinCH). East Midlands Research into Ageing Network (EMRAN) Discussion Paper Series. 2019. 24. www. nottingham.ac.uk/emran/documents/issue-25-emran-feb-2019.pdf

10 NICE. Falls in older people: assessing risk and prevention. 2013. www.nice.org.uk/guidance/cg161/resources/falls-in-older-peopleassessing-risk-and-prevention-pdf-35109686728645

11 Hoffmann TC, Glasziou PP, Boutron I, et al. Better reporting of interventions: template for intervention description and replication (TIDieR) checklist and guide. BMJ 2014;348:g1687. doi: 10.1136/ bmj.g1687

12 Robinson K, Allen F, Darby J, et al. Contamination in complex healthcare trials: the falls in care homes (FinCH) study experience. BMC Med Res Methodol 2020;20:46. doi: 10.1186/s12874-02000925-Z

13 Devlin NJ, Shah KK, Feng Y, Mulhern B, van Hout B. Valuing healthrelated quality of life: An EQ-5D-5L value set for England. Health Econ 2018:27:7-22. doi: 10.1002/hec 3564

14 Smith SC, Lamping DL, Banerjee S, et al. Development of a new measure of health-related quality of life for people with dementia: DEMQOL. Psychol Med 2007;37:737-46. doi: 10.1017/ S0033291706009469

15 Wade DT, Collin C. The Barthel ADL Index: a standard measure of physical disability? Int Disabil Stud 1988;10:64-7. doi: 10.3109/09638288809164105

16 Whitney J, Jackson SHD, Close JCT, Lord SR. Development and validation of a fall-related impulsive behaviour scale for residential care. Age Ageing 2013;42:754-8. doi: 10.1093/ageing/aft130

17 Whitney J, Close JC, Lord SR, Jackson SH. Identification of high risk fallers among older people living in residential care facilities: a simple screen based on easily collectable measures. Arch Gerontol Geriatr 2012;55:690-5. doi: 10.1016/j.archger.2012.05.010

18 van Hout B, Janssen MF, Feng YS, et al. Interim scoring for the EQ-5D-5L: mapping the EQ-5D-5L to EQ-5D-3L value sets. Value Health 2012;15:708-15. doi: 10.1016/j.jval.2012.02.008

19 Mulhern B, Rowen D, Brazier J, et al. Development of DEMQOL-U and DEMQOL-PROXY-U: generation of preference-based indices from DEMQOL and DEMQOL-PROXY for use in economic evaluation. Health Technol Assess 2013;17:v-xv, 1-140. doi: 10.3310/hta17050
20 White IR, Royston P, Wood AM. Multiple imputation using chained equations: Issues and guidance for practice. Stat Med 2011;30:37799. doi: $10.1002 / \operatorname{sim} .4067$

21 Rubin D, Schenker N. Multiple imputation for interval estimation from simple random samples with ignorable nonresponse. J Am Stat Assoc 1986:81:366-74 doi: 10.1080/01621459.1986.10478280

22 Corp S. STATA MP 16. www.stata-uk.com/software/stata. $\mathrm{html} /$ ?creative $=481207134673 \&$ keyword=stata\&matchtype $=$ p\&network $=g \&$ device $=c \&$ utm medium $=$ adwords\&utm campaign $=$ \&utm_source=\&gclid=CjOKCQiArvX_BRCyARIsAKsnTxOKddzliCfuOY XZLuOZjckYLh4uJ1Hk8laOZkJTCNP8j8OUmhtwGHoaAgOEEALw_wcB

23 R Core Team. R: A language and environment for statistical computing. R Foundation for Statistical Computing. 2020. wwwRprojectorg/. Vienna, Austria.

24 Involve. The UK standards for public involvement in research. 2019. https://sites.google.com/nihr.ac.uk/pi-standards/home

25 Staniszewska S, Brett J, Simera I, et al. GRIPP2 reporting checklists: tools to improve reporting of patient and public involvement in research. Res Involv Engagem 2017;3:13. doi: 10.1186/s40900017-0062-2

26 Spilsbury K, Hewitt C, Stirk L, Bowman C. The relationship between nurse staffing and quality of care in nursing homes: a systematic review. Int J Nurs Stud 2011;48:732-50. doi: 10.1016/j. ijnurstu.2011.02.014

27 Luff R, Laybourne A, Ferreira Z, et al. A guide to research with care homes. Qual Ageing Older Adults 2015:16:186-94 doi: 10.1108/ QAOA-06-2015-0027

28 Bunn F, Goodman C, Corazzini K, et al. Setting Priorities to Inform Assessment of Care Homes' Readiness to Participate in Healthcare Innovation: A Systematic Mapping Review and Consensus Process. Int J Environ Res Public Health 2020;17:987. doi: 10.3390/ ijerph17030987

29 Devi R, Chadborn NH, Meyer J, et al. How quality improvement collaboratives work to improve healthcare in care homes: a realist evaluation. Age Ageing 2021;50:1371-81. doi: 10.1093/ageing/ afab007

30 Meads DM, Martin A, Griffiths A. Cost-Effectiveness of Dementia Care Mapping in Care-Home Settings: Evaluation of a Randomised Controlled Trial. Appl Health Econ Health Policy 2020;18:237-47. doi: 10.1007/s40258-019-00531-1

31 Martin A, Meads D, Griffiths AW, Surr CA. How Should We Capture Health State Utility in Dementia? Comparisons of DEMQOLProxy-U and of Self- and Proxy-Completed EQ-5D-5L. Value Health 2019;22:1417-26. doi: 10.1016/j.jval.2019.07.002

32 Usman A, Lewis S, Hinsliff-Smith K, et al. Measuring health-related quality of life of care home residents: comparison of self-report with staff proxy responses. Age Ageing 2019;48:407-13. doi: 10.1093/ ageing/afy191

33 Chadborn N, Devi R, Hinsliff-Smith K, Banerjee J, Gordon A Quality improvement in long-term care settings: a scoping review of effective strategies used in care homes. Eur Geriatr Med 2021;12:17-26. doi: 10.1007/s41999-020-00389-w

34 Schols J, Gordon A. Residential and nursing home care: from the pas to the future. Michel JP, Beattie BL, Martin FC, Walston JDeds. Oxford Textbook of Geriatric Medicine. 2017.

35 Achterberg WP, Everink IH, van der Steen JT, Gordon AL. We're all different and we're the same: the story of the European nursing home resident. Age Ageing 2019;49:3-4. doi: 10.1093/ageing/afz145

Supplementary information: Additional material 